\title{
Electromagnetic wave propagation through a dielectric-chiral interface and through a chiral slab
}

\author{
S. Bassiri \\ Tracking Systems and Applications Section, Jet Propulsion Laboratory, California Institute of Technology, \\ Pasadena, California 91109 \\ C. H. Papas \\ Department of Electrical Engineering, California Institute of Technology, Pasadena, California 91125
}

N. Engheta

The Moore School of Electrical Engineering, University of Pennsylvania, Philadelphia, Pennsylvania 19104

Received September 15, 1987; accepted March 2, 1988

\begin{abstract}
The reflection from and transmission through a semi-infinite chiral medium are analyzed by obtaining the Fresnel equations in terms of parallel- and perpendicular-polarized modes, and a comparison is made with results reported previously. The chiral medium is described electromagnetically by the constitutive relations $\mathbf{D}=\epsilon \mathbf{E}+i \gamma \mathbf{B}$ and $\mathbf{H}=$ $i \gamma \mathbf{E}+(1 / \mu) \mathbf{B}$. The constants $\epsilon, \mu$, and $\gamma$ are real and have values that are fixed by the size, the shape, and the spatial distribution of the elements that collectively compose the medium. The conditions are obtained for the total internal reflection of the incident wave from the interface and for the existence of the Brewster angle. The effects of the chirality on the polarization and the intensity of the reflected wave from the chiral half-space are discussed and illustrated by using the Stokes parameters. The propagation of electromagnetic waves through an infinite slab of chiral medium is formulated for oblique incidence and solved analytically for the case of normal incidence.
\end{abstract}

\section{INTRODUCTION}

In this paper we present a theoretical study of the plane waves in chiral media. In particular, the problem of reflection from and transmission through a dielectric-chiral interface and wave propagation in an infinite chiral slab is discussed in detail. The motivation for this study, beside its theoretical importance, is provided by its applicability to the problem of vegetation layers in remote sensing. Since certain types of terrestrial vegetation layer can be thought of as chiral media, the analytical results of this paper provide the necessary tools for analyzing and interpreting the experimental data.

A chiral medium is a macroscopically continuous medium composed of equivalent chiral objects that are uniformly distributed and randomly oriented. A chiral object is a three-dimensional body that cannot be brought into congruence with its mirror image by translation and rotation. An object of this sort has the property of handedness and must be either left-handed or right-handed. An object that is not chiral is said to be achiral. Thus all objects are either chiral or achiral. Some chiral objects occur naturally in two versions related to each other as a chiral object and its mirror image. Objects so related are said to be enantiomorphs of each other. If a chiral object is left-handed, its enantiomorph is right-handed, and vice versa. An example of a chiral object is the wire helix; other simple oxamples are the Möbius strip and the irregular tetrahedron.

When a linearly polarized wave is incident normally upon a slab of chiral medium, two waves are generated in the medium; one is a left-circularly polarized wave and the other is a right-circularly polarized wave of a different phase velocity. Behind the slab the two waves combine to yield a linearly polarized wave whose plane of polarization is rotated with respect to the plane of polarization of the incident wave. The amount of rotation depends on the distance traveled in the medium, and this implies that the optical activity occurs not at the surfaces of the slab but throughout the medium. Optical activity in a chiral medium differs from the phenomenon of Faraday rotation in, say, a magnetically biased plasma by the fact that the former is independent of the direction of propagation whereas the latter is not. The optical activity is invariant under time reversal, and the Faraday rotation is invariant under spatial inversion.

The phenomenon of optical activity was first discovered by Arago ${ }^{1}$ in 1811 . He found that crystals of quartz rotate the plane of polarization of linearly polarized light that is transmitted in the direction of its optical axis. The experiments of Biot, ${ }^{2-4}$ dating from 1812 to 1838 , on plates of quartz established (1) the dependence of optical activity on the thickness of the plate, (2) the unequal rotations of the planes of polarization of light of different wavelengths, and (3) the absence of any optical activity when two plates of quartz of the same thickness but opposite handedness are used. In 1815 Biot $^{5}$ discovered that optical activity is not restricted to crystalline solids but appears as well in other media, such as oils of turpentine and laurel and aqueous solutions of tartaric acid. Fresnel ${ }^{6}$ showed in 1822 that a ray of light traveling along the axis of a crystal of quartz is resolved into two circularly polarized rays of opposite handed- 
nesses that travel with unequal phase velocities. He argued that the difference in the two wave velocities is the cause of optical activity. In $1848 \mathrm{Pasteur}^{7}$ postulated that molecules are three-dimensional figures and that the optical activity of a medium is caused by the chirality of its molecules. More recently, Lindman ${ }^{8,9}$ (in 1920 and 1922) and Pickering ${ }^{10}$ (in 1945) devised a macroscopic model for the phenomenon by using microwaves instead of light and using wire spirals instead of chiral molecules. They illustrated the molecular process responsible for optical activity by using this model. Many other experiments were performed, and a thorough account of them is contained in a book by Lowry. ${ }^{11}$

By the end of the 19th century experimental and empirical facts on optical activity were well established, and physicists had started to develop theories in order to explain the interaction of electromagnetic waves with chiral media. About 1915, Born, ${ }^{12}$ Oseen, ${ }^{13}$ and Gray ${ }^{14}$ put forward independently and almost simultaneously the explanation of optical activity for a particular molecular model. The molecular model used was that of a spatial distribution of coupled oscillators. Kuhn ${ }^{15}$ also contributed greatly to solution of the problem by considering the simplest case of the coupledoscillator model to show optical activity. In 1937 Condon et $a l .{ }^{16}$ showed that it is possible to explain optical activity by considering a single oscillator moving in a dissymmetric field. A detailed account of these microscopic theories was given by Condon. ${ }^{17}$ More-recent work includes several papers by Bohren examining the reflection of electromagnetic waves from chiral spheres and cylinders, ${ }^{18,19}$ a paper on light reflection from chiral surfaces by Bokut and Federov, ${ }^{20}$ and the book by Kong ${ }^{21}$ and numerous references therein regarding general bianisotropic media. Shortly thereafter, the macroscopic treatment of the interaction of electromagnetic waves with chiral structures, which is the theoretical counterpart of Lindman's experiments, was given by Jaggard et $a l .22$ In their paper the interaction of electromagnetic waves with a collection of randomly oriented short metallic helices of the same handedness was studied, and the optical activity in such media was placed in evidence. The most recent work includes that on transition radiation at a chiral-achiral interface by Engheta and Mickelson, ${ }^{23}$ on the reflection of waves from a chiral-achiral interface by Silverman ${ }^{24,25}$ and Lakhtakia et $a l .,{ }^{26}$ on the scattering of electromagnetic waves from nonspherical chiral objects by Lakhtakia et al. ${ }^{27}$ on light propagation through an infinite chiral medium by Silverman and Sohn, ${ }^{28}$ and on the dyadic Green's function and the dipole radiation for an unbounded, isotropic, lossless chiral medium by Bassiri et al. ${ }^{29,30}$

Silverman ${ }^{24}$ recently derived the Fresnel amplitudes in terms of the right- and left-circularly polarized modes of a chiral medium. He described the chiral medium by two sets of symmetric and asymmetric constitutive relations. The symmetric set of constitutive relations described by Silverman is physically equivalent to the set used in this paper and can be shown to produce physically identical results. In the present paper, the problem of reflection from and transmission through a semi-infinite chiral medium is revisited and analyzed in terms of parallell- and perpendicular-polarized modes for the set of constitutive relations introduced in Ref. 22. The Fresnel reflection and transmission amplitudes are shown to be physically equivalent to those given in Ref. 24 for the symmetric constitutive relations. In addition, the conditions for the total internal reflection of the incident wave from the interface and for the existence of the Brewster angle are obtained. The propagation of waves through an infinite slab of chiral medium is also analyzed. Finally, the conclusions and brief applications of these analyses are given.

For a chiral medium composed of lossless, reciprocal, short wire helices that are all of the same handedness, the constitutive relations for time-harmonic fields $[\exp (-i \omega t)]$ have the form $\mathbf{D}=\epsilon \mathbf{E}+i \gamma \mathbf{B}$, and $\mathbf{H}=i \gamma \mathbf{E}+(1 / \mu) \mathbf{B}$, where $\epsilon$, $\mu$, and $\gamma$ are real quantities. Moreover, it was conjectured ${ }^{22}$ that these constitutive relations apply not only to chiral media composed of helices but also to lossless, reciprocal, isotropic, chiral media composed of chiral objects of arbitrary shape. Since $\mathbf{D}$ and $\mathbf{E}$ are polar vectors and $\mathbf{B}$ and $\mathbf{H}$ are axial vectors, it follows that $\epsilon$ and $\mu$ are true scalars and $\gamma$ is a pseudoscalar.

This means that, when the axes of a right-handed Cartesian coordinate system are reversed to form a left-handed Cartesian coordinate system, $\gamma$ changes in sign, whereas $\epsilon$ and $\mu$ remain unchanged. Thus the handedness of the medium is manifested by the quantity $\gamma$. When $\gamma>0$, the medium is right-handed and the sense of polarization is right-handed; when $\gamma<0$, the medium is left-handed and the sense of polarization is left-handed; and when $\gamma=0$, the medium is simple, and there is no optical activity.

When $(1 / i \omega) \nabla \times \mathbf{E}$ is substituted for $\mathbf{B}$ in the first constitutive relation, it is evident that the value of $\mathbf{D}$ at any given point in space depends not only on the value of $\mathbf{E}$ at that particular point but also on the behavior of $\mathbf{E}$ in the vicinity of this point; that is, $\mathbf{D}$ depends also on the derivatives of E. ${ }^{31}$ This nonlocal spatial relationship between $\mathbf{D}$ and $\mathbf{E}$ is called spatial dispersion. Therefore the medium described by the above-mentioned constitutive relations is a spatially dispersive, isotropic, lossless, reciprocal, chiral medium.

\section{SEMI-INFINITE CHIRAL MEDIA}

\section{Laws of Reflection and Refraction}

When a plane wave is incident upon a boundary between a dielectric and a chiral medium, it splits into two transmitted waves proceeding into the chiral medium and a reflected wave propagating back into the dielectric. To formulate mathematically the problem of reflection from and transmission through a semi-infinite chiral medium, a Cartesian coordinate system $(x, y, z)$ is introduced. The unit vectors of this coordinate system are denoted by $\hat{e}_{x}, \hat{e}_{y}$, and $\hat{e}_{z}$. As shown in Fig. 1, the $x y$ plane is the plane of interface of a simple homogeneous dielectric with permittivity $\epsilon_{1}$, permeability $\mu_{1}$, and a homogeneous chiral medium described by the constitutive relations

$$
\begin{aligned}
& \mathbf{D}=\epsilon \mathbf{E}+i \gamma \mathbf{B}, \\
& \mathbf{H}=i \gamma \mathbf{E}+(1 / \mu) \mathbf{B} .
\end{aligned}
$$

Inside the chiral medium there are two modes of propagation: a right-circularly polarized wave of phase velocity $\omega / h_{1}$ and a left-circularly polarized wave of phase velocity $\omega / h_{2}$. The wave numbers $h_{1}$ and $h_{2}$ are given by ${ }^{30}$

$$
h_{1}=\omega \mu \gamma+\left(\omega^{2} \mu^{2} \gamma^{2}+k^{2}\right)^{1 / 2}
$$




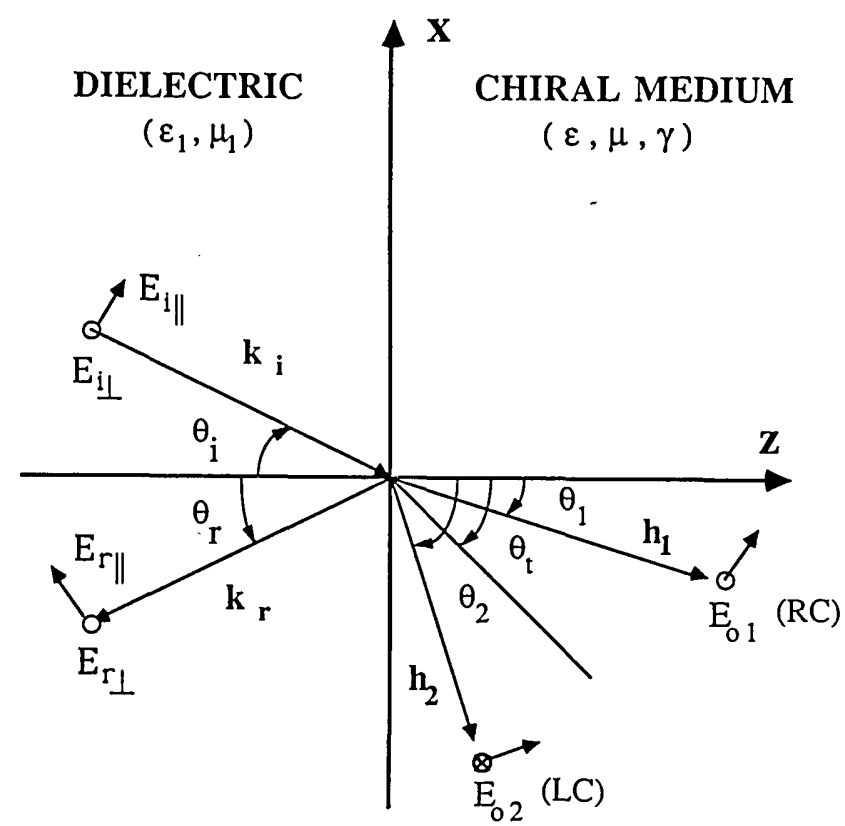

Fig. 1. Orientation of the wave vectors of the incident wave, the reflected wave, and the transmitted waves at an oblique incidence on a semi-infinite chiral medium. In the chiral medium the $h_{1}$ and the $h_{2}$ waves are right-circularly (RC) and left-circularly (LC) polarized waves, respectively. The two angles of refraction are denoted by $\theta_{1}$ and $\theta_{2}$. For $\gamma=0$, these two angle approach $\theta_{t}$.

$$
h_{2}=-\omega \mu \gamma+\left(\omega^{2} \mu^{2} \gamma^{2}+k^{2}\right)^{1 / 2} \text {, }
$$

where $k^{2}=\omega^{2} \mu \epsilon$ and where $\epsilon$ and $\mu$ are the permittivity and the permeability of the chiral medium, respectively. For $\gamma$ $>0$ the right-circularly polarized wave is the slower mode, whereas for $\gamma<0$ the left-circularly polarized wave is the slower mode.

It is assumed that a monochromatic plane wave is obliquely incident upon the interface. The complex-constant amplitude vectors of the incident, reflected, and transmitted plane waves always lie on planes perpendicular to the direction of their propagation. Therefore it is always possible to decompose any one of these amplitudes into a component normal to the plane of incidence and a second component lying in the plane of incidence ( $x z$ plane), as shown in Fig. 1. The plane of incidence is the plane containing the normal to the interface and the wave vector of the incident wave.

From the boundary conditions, that is, the continuity of the tangential electric field and the tangential magnetic field at the interface, it can be shown that

$$
\mathbf{k}_{i} \times \hat{e}_{z}=\mathbf{k}_{r} \times \hat{e}_{z}=\mathbf{h}_{1} \times \hat{e}_{z}=\mathbf{h}_{2} \times \hat{e}_{z},
$$

where $\mathbf{k}_{i}, \mathbf{k}_{\boldsymbol{r}}, \mathbf{h}_{1}$, and $\mathbf{h}_{2}$ are the wave vectors of the incident wave, the reflected wave, and the two transmitted waves, respectively. ${ }^{32}$ When the magnitude of the vector Eq. (5) is taken it is found that

$$
k_{i} \sin \theta_{i}=k_{r} \sin \theta_{r}=h_{1} \sin \theta_{1}=h_{2} \sin \theta_{2} ;
$$

since $k_{i}=k_{r}$, then $\theta_{i}=\theta_{r}$. From Eq. (6), the angles $\theta_{1}$ and $\theta_{2}$ corresponding to the two transmitted waves (see Fig. 1) are found to be

$$
\theta_{1}=\arcsin \left(\frac{k_{i} \sin \theta_{i}}{h_{1}}\right)
$$

$$
\theta_{2}=\arcsin \left(\frac{k_{i} \sin \theta_{i}}{h_{2}}\right),
$$

where $\theta_{i}$ is the angle of incidence, $k_{i}=\omega\left(\mu_{1} \epsilon_{1}\right)^{1 / 2}$, and $h_{1}$ and $h_{2}$ are given by Eqs. (3) and (4). When $\gamma=0$, the angle of the transmitted wave is given by

$$
\theta_{t}=\arcsin \left(\frac{k_{i} \sin \theta_{i}}{k}\right)
$$

where $\theta_{t}$ is the refraction angle of a dielectric-dielectric interface. Therefore, as $\gamma \rightarrow 0$, angles $\theta_{1}$ and $\theta_{2}$ approach $\theta_{t}$. As $\gamma \rightarrow \infty$, angles $\theta_{1}$ and $\theta_{2}$ approach 0 and $\pi / 2$, respectively; that is, the $h_{2}$ wave becomes evanescent and only the $h_{1}$ wave propagates, and its direction of propagation is along the positive $z$ axis. As $\gamma \rightarrow-\infty$, angles $\theta_{1}$ and $\theta_{2}$ approach $\pi / 2$ and 0 , respectively. In this case the $h_{1}$ wave becomes evanescent and the $h_{2}$ wave propagates along the positive $z$ axis.

\section{Total Internal Reflection}

In general, there can be two propagating transmitted waves inside the chiral medium, namely, the $h_{1}$ wave and the $h_{2}$ wave. When neither of these waves propagates inside the chiral medium, the phenomenon of total internal reflection occurs. By letting $\theta_{1}$ and $\theta_{2}$ be equal to $\pi / 2$ in Eqs. (7) and (8), the critical angles of incidence $\theta_{c 1}$ and $\theta_{c 2}$ can be found:

$$
\begin{aligned}
& \theta_{c 1}=\arcsin \left[\frac{\mu \gamma+\left(\mu^{2} \gamma^{2}+\mu \epsilon\right)^{1 / 2}}{\left(\mu_{1} \epsilon_{1}\right)^{1 / 2}}\right], \\
& \theta_{c 2}=\arcsin \left[\frac{-\mu \gamma+\left(\mu^{2} \gamma^{2}+\mu \epsilon\right)^{1 / 2}}{\left(\mu_{1} \epsilon_{1}\right)^{1 / 2}}\right] .
\end{aligned}
$$

When $\gamma>0$ and $h_{2}<h_{1}<k_{i}$, then $\theta_{c 1}$ is always greater than $\theta_{c 2}$. There are three possibilities:

(1) If $\theta_{i}<\theta_{c 2}<\theta_{c 1}$, then both $h_{1}$ and $h_{2}$ waves propagate. Their directions of propagation are given by Eqs. (7) and (8).

(2) If $\theta_{c 2} \leq \theta_{i}<\theta_{c 1}$, then only the $h_{1}$ wave propagates, and the $h_{2}$ wave becomes evanescent. The direction of propagation for the $h_{1}$ wave is given by Eq. (7).

(3) If $\theta_{c 2}<\theta_{c 1} \leq \theta_{i}$, then neither of the waves propagates and there is total internal reflection of the two waves into the dielectric.

When $\gamma<0$ and $h_{1}<h_{2}<k_{i}$, then $\theta_{c 1}$ is always less than $\theta_{c 2}$. There are three possibilities:

(1) If $\theta_{i}<\theta_{c 1}<\theta_{c 2}$, then both $h_{1}$ and $h_{2}$ waves propagate. The directions of propagation of these waves are given by Eqs. (7) and (8).

(2) If $\theta_{c 1} \leq \theta_{i}<\theta_{c 2}$, then only the $h_{2}$ wave propagates, and the $h_{1}$ wave becomes evanescent. The direction of propagation for the $h_{2}$ wave is given by Eq. (8).

(3) If $\theta_{c 1}<\theta_{c 2} \leq \theta_{i}$, then neither of the waves propagates, and the phenomenon of total internal reflection occurs.

Obviously, there are other possibilities. For example, when $\gamma>0$ and $h_{2} \leqslant k_{i} \leqslant h_{1}$ or when $\gamma \leqslant 0$ and $h_{1} \leqslant h_{i} \leqslant h_{2}$, there is only one real solution for the critical angle of incidence.

Depending on the incidence angle and the relative values 
of $h_{1}, h_{2}$, and $k_{i}$, then one, both, or none of the transmitted waves propagates inside the chiral medium.

\section{Fresnel Equations}

In order to study the power carried by the reflected and transmitted waves and also the polarization properties of these waves, it is necessary to determine the complex-constant amplitude vectors associated with these waves. This is done by matching the fields at the interface, using the boundary conditions ${ }^{33}$

$$
\begin{aligned}
\left(\mathbf{E}_{0 i}+\mathbf{E}_{0 r}\right) \times \hat{e}_{z} & =\left(\mathbf{E}_{01}+\mathbf{E}_{02}\right) \times \hat{e}_{z}, \\
\left(\mathbf{H}_{0 i}+\mathbf{H}_{0 r}\right) \times \hat{e}_{z} & =\left(\mathbf{H}_{01}+\mathbf{H}_{02}\right) \times \hat{e}_{z},
\end{aligned}
$$

The expressions for $E_{r \perp}, E_{r \|}, E_{01}$, and $E_{02}$ in terms of the components of the incident wave can be written as

$$
\left(\begin{array}{c}
E_{r \perp} \\
E_{r \|}
\end{array}\right)=\left[\begin{array}{ll}
R_{11} & R_{12} \\
R_{21} & R_{22}
\end{array}\right]\left(\begin{array}{c}
E_{i \perp} \\
E_{i \|}
\end{array}\right)
$$

and

$$
\left(\begin{array}{l}
E_{01} \\
E_{02}
\end{array}\right)=\left[\begin{array}{ll}
T_{11} & T_{12} \\
T_{21} & T_{22}
\end{array}\right]\left(\begin{array}{l}
E_{i \perp} \\
E_{i \|}
\end{array}\right)
$$

where the $2 \times 2$ matrix in Eq. (14) is the reflection coefficient matrix and its entries are

$$
\begin{aligned}
& R_{11}=\frac{\cos \theta_{i}\left(1-g^{2}\right)\left(\cos \theta_{1}+\cos \theta_{2}\right)+2 g\left(\cos ^{2} \theta_{i}-\cos \theta_{1} \cos \theta_{2}\right)}{\cos \theta_{i}\left(1+g^{2}\right)\left(\cos \theta_{1}+\cos \theta_{2}\right)+2 g\left(\cos ^{2} \theta_{i}+\cos \theta_{1} \cos \theta_{2}\right)}, \\
& R_{12}=\frac{-2 i g \cos \theta_{i}\left(\cos \theta_{1}-\cos \theta_{2}\right)}{\cos \theta_{i}\left(1+g^{2}\right)\left(\cos \theta_{1}+\cos \theta_{2}\right)+2 g\left(\cos ^{2} \theta_{i}+\cos \theta_{1} \cos \theta_{2}\right)}, \\
& R_{21}=\frac{-2 i g \cos \theta_{i}\left(\cos \theta_{1}-\cos \theta_{2}\right)}{\cos \theta_{i}\left(1+g^{2}\right)\left(\cos \theta_{1}+\cos \theta_{2}\right)+2 g\left(\cos ^{2} \theta_{i}+\cos \theta_{1} \cos \theta_{2}\right)}, \\
& R_{22}=\frac{\cos \theta_{i}\left(1-g^{2}\right)\left(\cos \theta_{1}+\cos \theta_{2}\right)-2 g\left(\cos ^{2} \theta_{i}-\cos \theta_{1} \cos \theta_{2}\right)}{\cos \theta_{i}\left(1+g^{2}\right)\left(\cos \theta_{1}+\cos \theta_{2}\right)+2 g\left(\cos ^{2} \theta_{i}+\cos \theta_{1} \cos \theta_{2}\right)},
\end{aligned}
$$

where $\mathbf{E}_{0 i}$ and $\mathbf{E}_{0 r}$ are the complex-constant amplitudes of the incident and reflected electric fields, respectively. Simi- where $g=\left[\left(\mu_{1} / \epsilon_{1}\right) \gamma^{2}+\left(\mu_{1} \epsilon / \epsilon_{1} \mu\right)\right]^{1 / 2}$. The $2 \times 2$ matrix in Eq. (15) is the transmission coefficient matrix, and its entries are

$$
\begin{aligned}
& T_{11}=\frac{-2 i \cos \theta_{i}\left(g \cos \theta_{i}+\cos \theta_{2}\right)}{\cos \theta_{i}\left(1+g^{2}\right)\left(\cos \theta_{1}+\cos \theta_{2}\right)+2 g\left(\cos ^{2} \theta_{i}+\cos \theta_{1} \cos \theta_{2}\right)}, \\
& T_{12}=\frac{2 \cos \theta_{i}\left(\cos \theta_{i}+g \cos \theta_{2}\right)}{\cos \theta_{i}\left(1+g^{2}\right)\left(\cos \theta_{1}+\cos \theta_{2}\right)+2 g\left(\cos ^{2} \theta_{i}+\cos \theta_{1} \cos \theta_{2}\right)}, \\
& T_{21}=\frac{2 i \cos \theta_{i}\left(g \cos \theta_{i}+\cos \theta_{1}\right)}{\cos \theta_{i}\left(1+g^{2}\right)\left(\cos \theta_{1}+\cos \theta_{2}\right)+2 g\left(\cos ^{2} \theta_{i}+\cos \theta_{1} \cos \theta_{2}\right)}, \\
& T_{22}=\frac{2 \cos \theta_{i}\left(\cos \theta_{i}+g \cos \theta_{1}\right)}{\cos \theta_{i}\left(1+g^{2}\right)\left(\cos \theta_{1}+\cos \theta_{2}\right)+2 g\left(\cos ^{2} \theta_{i}+\cos \theta_{1} \cos \theta_{2}\right)} .
\end{aligned}
$$

larly, $\mathbf{E}_{01}$ and $\mathbf{E}_{02}$ are the amplitudes of the electric fields associated with the right-circularly and left-circularly polarized transmitted waves, respectively (see Fig. 1).

It is assumed that the amplitude, the polarization, the direction of propagation, and the frequency of the incident field are known. To find the complex-constant amplitude vectors of the reflected and transmitted waves, the boundary conditions at the interface must be applied to the $x$ and $y$ components of the electric and magnetic fields. A system of four nonhomogeneous equations with the four unknowns $E_{r \perp}, E_{r \|}, E_{01}$, and $E_{02}$ is obtained. The subscript $\perp$ refers to the amplitude of the field component that is perpendicular to the plane of incidence, and the subscript \| refers to the amplitude of the field component that lies in the plane of incidence.
When the incident wave falls normally upon the interface, that is, $\theta_{i}=0$, Eqs. (16)-(23) reduce to

$$
\begin{aligned}
& R_{11}=R_{22}=\frac{1-g}{1+g}, \\
& R_{12}=R_{21}=0 \\
& T_{11}=-i T_{22}=\frac{-i}{1+g}, \\
& T_{12}=-i T_{21}=\frac{1}{1+g} .
\end{aligned}
$$

When the Fresnel reflection and transmission amplitudes above, which are obtained by using parallel- and perpendicular- 
polarized modes and the constitutive relations given by Eqs. (1) and (2), are compared with those obtained by Silverman, ${ }^{24}$ which are calculated by using the right-circularly and left-circularly polarized modes and the other symmetric constitutive relations, the physical equivalence between the two sets of amplitudes is evident. This supports the fact that the two sets of symmetric constitutive relations lead to physically equivalent results.

\section{Brewster Angle}

Under a certain condition a monochromatic plane wave of arbitrary polarization, on reflection from a chiral medium, becomes a linearly polarized wave. The angle of incidence that satisfies this condition is called the Brewster angle. The plane containing the electric field vector and the direction of propagation is called the plane of polarization. For a linearly polarized wave, the angle between the plane of polarization and the plane of incidence is called the azimuthal angle. This angle lies in the range from $-\pi / 2$ to $\pi / 2$. It is defined to be positive whenever the sense of rotation of the plane of polarization toward the plane of incidence and the direction of wave propagation form a right-handed screw. Let $\alpha_{i}$ and $\alpha_{r}$ be the azimuthal angles of the incident and reflected waves, respectively. From the above definitions we can write

$$
\begin{aligned}
& \tan \alpha_{i}=\frac{E_{i \perp}}{E_{i \|}}, \\
& \tan \alpha_{r}=\frac{E_{r \perp}}{E_{r \|}},
\end{aligned}
$$

where $\alpha_{i}$ and $\alpha_{r}$ can be complex angles. The amplitudes of the perpendicular and parallel components of the incident and reflected waves are related by the matrix equation (14). By using Eqs. (28), (29), and (14), it can be shown that

$$
\tan \alpha_{r}=\frac{R_{12}+R_{11} \tan \alpha_{i}}{R_{22}+R_{21} \tan \alpha_{i}} .
$$

If the incident wave is incident upon the interface at the Brewster angle $\theta_{\mathrm{B}}$, then the reflected wave must be linearly polarized. Therefore $\alpha_{r}$ must be a real constant for all $\alpha_{i}$ at this angle. ${ }^{34}$ When Eq. (30) is differentiated with respect to $\alpha_{i}$, the following condition is obtained:

$$
R_{11} R_{22}-R_{12} R_{21}=0 \text {. }
$$

Under this condition, Eq. (30) becomes

$$
\tan \alpha_{r}=\frac{R_{12}}{R_{22}}=\frac{R_{11}}{R_{21}} \text {. }
$$

When Eqs. (16)-(19) are substituted into Eq. (31) the following equation is obtained:

$$
\begin{aligned}
& \left(1-g^{2}\right)^{2} \cos ^{2} \theta_{i}\left(\cos \theta_{1}+\cos \theta_{2}\right)^{2} \\
& 4 g^{2}\left(\cos ^{2} \theta_{i}-\cos ^{2} \theta_{1}\right)\left(\cos ^{2} \theta_{i}-\cos ^{2} \theta_{2}\right),
\end{aligned}
$$

where $\theta_{1}$ and $\theta_{2}$ can be written in terms of $\theta_{i}$, using Eqs. (7) and (8). The incidence angle that satisfies the transcendental equation (33) is the Brewster angle, and it can be solved for by using standard numerical techniques.

\section{Stokes Parameters}

A plane monochromatic TEM wave of the form

$$
\mathbf{E}=\left(E_{\perp} \hat{e}_{\perp}+E_{\|} \hat{e}_{\|}\right) \exp (i \mathbf{k} \cdot \mathbf{r}-i \omega t)
$$

is by nature elliptically polarized. The Stokes parameters, ${ }^{33}$ which are defined by

$$
\begin{aligned}
& S_{0}=E_{\perp} E_{\perp}{ }^{*}+E_{\|} E_{\|}{ }^{*}, \\
& S_{1}=E_{\perp} E_{\perp}{ }^{*}-E_{\|} E_{\|}{ }^{*}, \\
& S_{2}=2 \operatorname{Re}\left\{E_{\perp} E_{\|}{ }^{*}\right\}, \\
& S_{3}=2 \operatorname{Im}\left\{E_{\perp} E_{\|}{ }^{*}\right\},
\end{aligned}
$$

describe completely the state of polarization of the wave. The power carried by the wave is proportional to $S_{0}$, and the orientation angle $\psi$ of the polarization ellipse, shown in Fig. 2 , is given by

$$
\tan 2 \psi=\frac{S_{2}}{S_{1}} \quad(0 \leq \psi \leq \pi),
$$

and the ellipticity angle $\chi$ is given by (see Fig. 2)

$$
\tan \chi=\frac{\left(S_{3} / S_{0}\right)}{1+\left[1-\left(S_{3} / S_{0}\right)^{2}\right]^{1 / 2}} \quad(-\pi / 4 \leq \chi \leq \pi / 4) .
$$

The numerical value of $\tan \chi$ yields the reciprocal of the axial ratio $a_{0} / b_{0}$ of the ellipse, where $a_{0}$ and $b_{0}$ are the semimajor and semiminor axes of the ellipse, respectively. The sign of $\chi$ differentiates the two senses of polarization, e.g., for left-handed polarization $0<\chi \leq \pi / 4$ and for right-handed polarization $-\pi / 4 \leq \chi<0$.

The power carried by the reflected wave can be found by substituting the expressions for $E_{r \perp}$ and $E_{r \|}$ into Eq. (35). These expressions can be found by using Eqs. (16)-(19). Since the analytical expression for the power involves many parameters and is not very informative, only the graphs of the reflected power versus the angle of incidence are given in Fig. 3. If the incident electric field is in the plane of incidence, then the normalized reflected power is denoted by $P_{\|}$; if it is perpendicular to the plane of incidence, then the normalized reflected power is denoted by $P_{\perp}$. The normal-

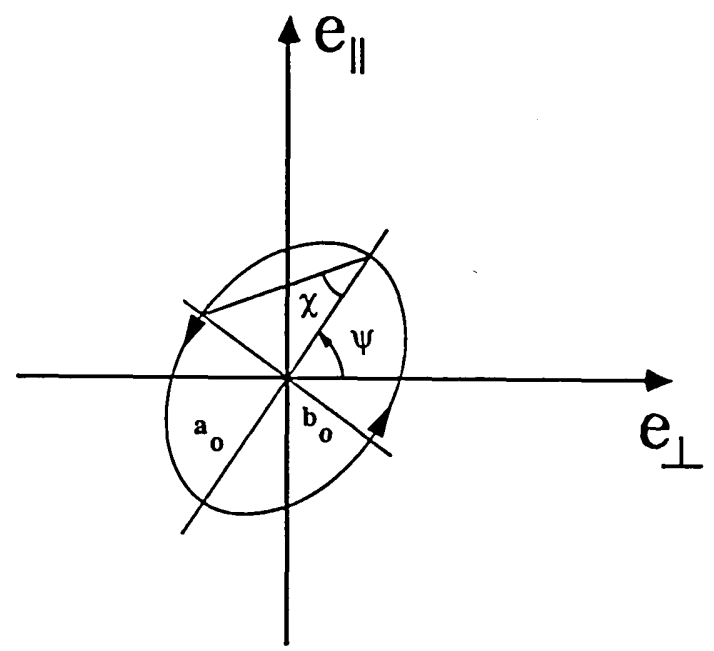

Fig. 2. Polarization ellipse for right-elliptically polarized wave having an orientation angle $\psi$ and an ellipticity angle $\chi$. 
Case (a)
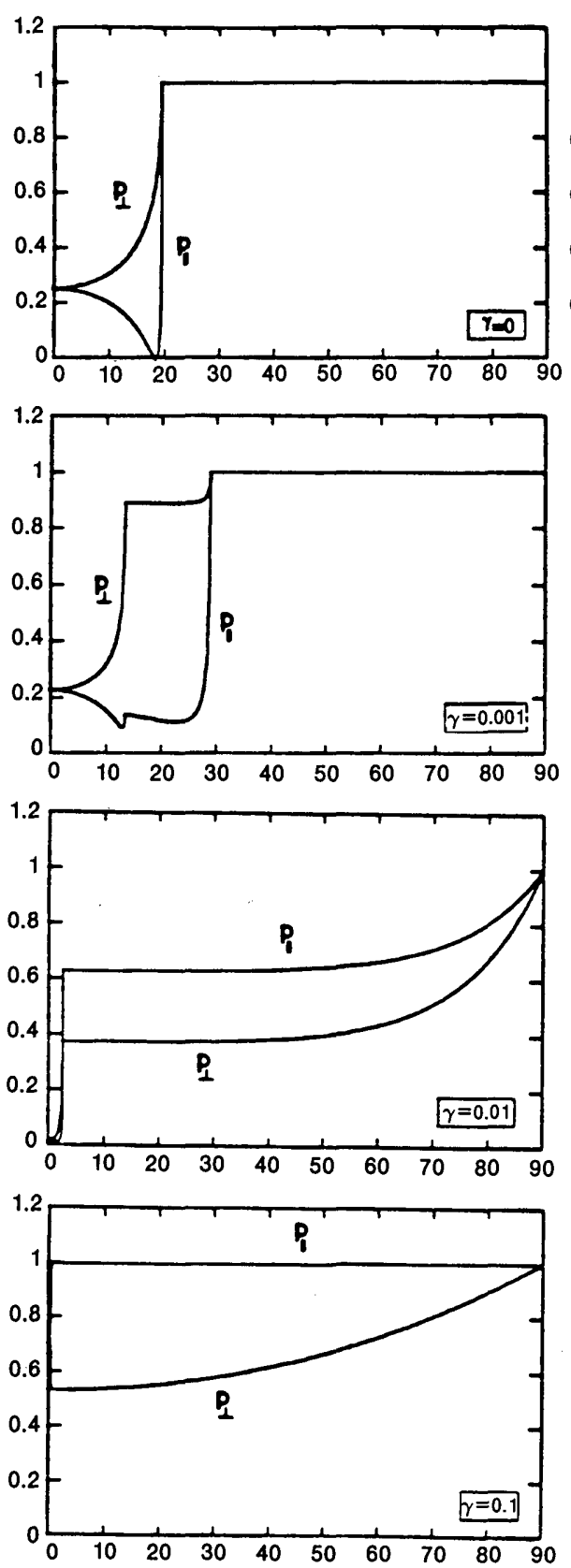

$\boldsymbol{\theta}_{i}$ (degree)

incidence angle
Case (b)
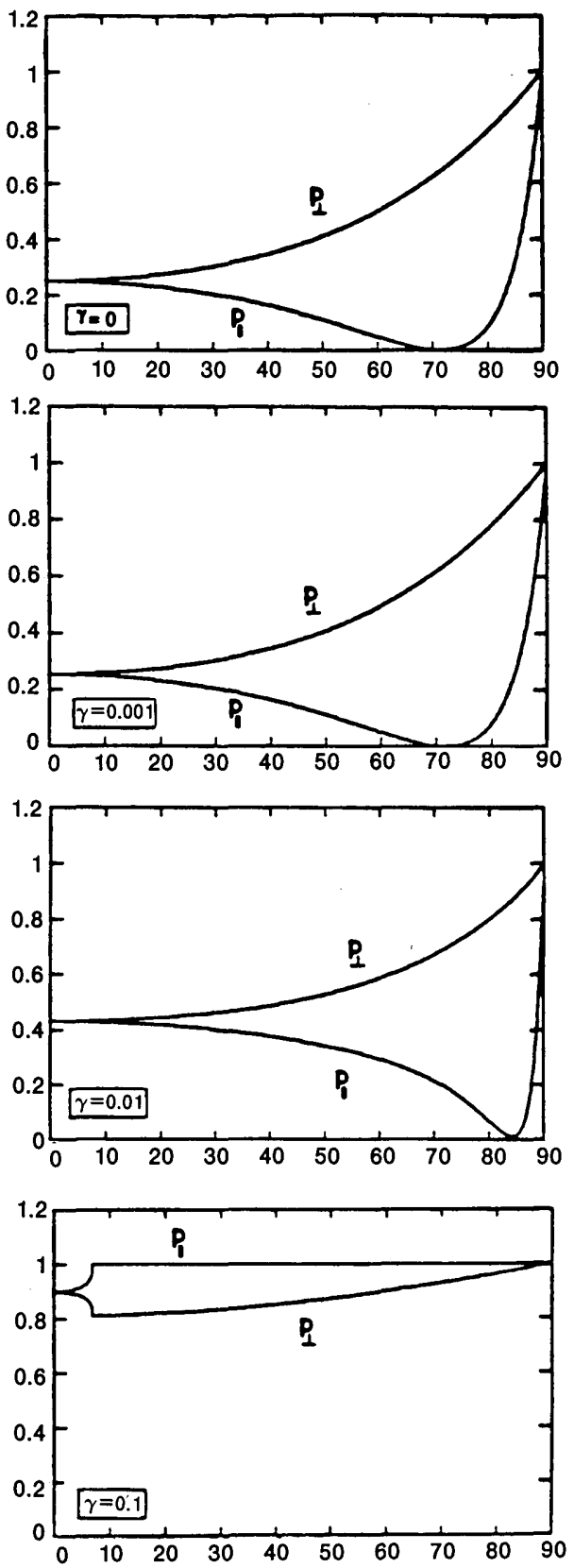

\section{$\theta_{i}$ (degree)}

incidence angle

Fig. 3. Normalized reflected power (vertical axes) as a function of the incident angle $\theta_{i}$ (horizontal axes). For Case (a), $\epsilon_{1}=9 \epsilon_{0}, \epsilon=\epsilon_{0} ;$ for Case (b), $\epsilon_{1}=\epsilon_{0}, \epsilon=9 \epsilon_{0}$. The values of $\gamma$ are shown on each plot. For both cases it is assumed that $\mu_{1}=\mu=\mu_{0}$.

ization is with respect to the power carried by the corresponding component of the incident wave. In Fig. 3 the normalized power is graphed versus the angle of incidence, as $\gamma$ varies, for two different cases, (a) and (b). The following conclusions can be drawn from the figures.

Case (a) $\epsilon<\epsilon_{1}$; the dielectric is denser than the chiral medium. In this case, there is a Brewster angle for small values of $\gamma$. For the parallel polarization of the incident field, the power $\left(P_{\|}\right)$is almost totally reflected when $\gamma$ is large, and as $\gamma$ increases, the normalized reflected power increases and approaches unity.

Case (b) $\epsilon>\epsilon_{1}$; the chiral medium is denser than the dielectric. In this case, there is a Brewster angle for parallel polarization of the incident field. As $\gamma$ increases, the Brew- 
ster angle approaches $90^{\circ}$ and then disappears, and $P_{\|}$becomes almost equal to unity; that is, most of the incident power is reflected back into the dielectric.

Polarization characteristics of the reflected wave can be obtained from Eqs. (39) and (40). By substituting the expressions for $E_{r \perp}$ and $E_{r \|}$ into Eq. (37), it may be shown that $S_{2}=0$. Hence

$$
\psi=\frac{1}{2} \arctan \left(\frac{S_{2}}{S_{1}}\right)=0 ;
$$

that is, the major and minor axes of the polarization ellipse are along $\hat{e}_{\perp}$ and $\hat{e}_{\|}$, respectively. The ellipticity $\left(b_{0} / a_{0}\right)$ of the ellipse can be found from Eq. (40) and is plotted versus the angle of incidence, for different values of $\gamma$, in Fig. 4. If the incident electric field is in the plane of incidence, then
Case (a)
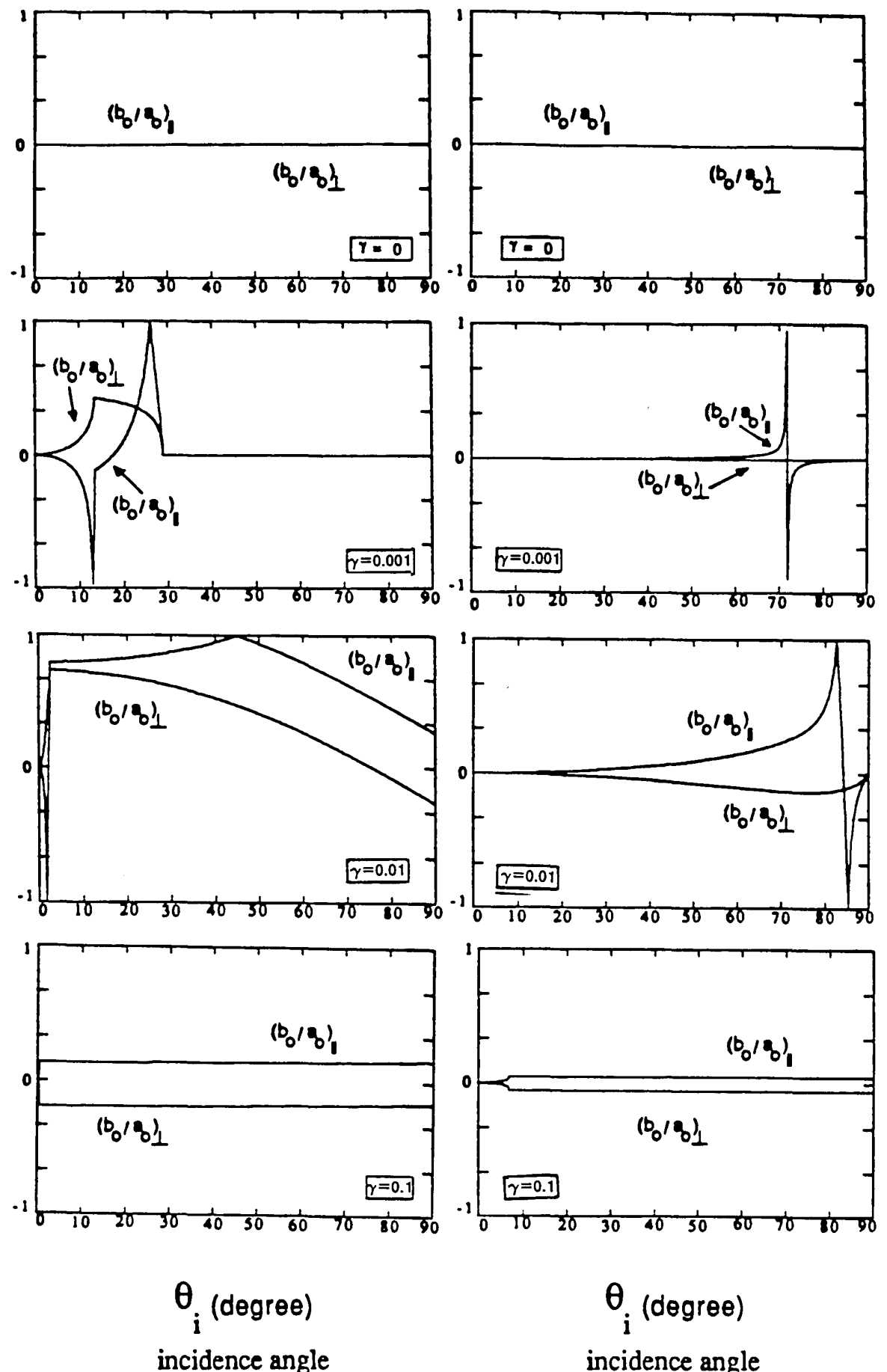

Case (b) . 
the ellipticity is denoted by $\left(b_{0} / a_{0}\right) \|$; if the incident electric field is perpendicular to the plane of incidence, then the ellipticity is denoted by $\left(b_{0} / a_{0}\right)_{\perp}$. Figure 4 shows $\left(b_{0} / a_{0}\right)$ for the two cases, (a) and (b). The following conclusions can be drawn from the figures.

Case (a) $\epsilon<\epsilon_{1}$; the dielectric is denser than the chiral medium. In this case, for small $\gamma$, there exists an angle of incidence for which a right-circularly polarized reflected wave can be observed when the incident electric field is polarized perpendicular to the plane of incidence. $\left(b_{0} / a_{0}\right)_{\|}$ and $\left(b_{0} / a_{0}\right)_{\perp}$ of the left- and right-elliptically polarized reflected waves, respectively, get thinner as $\gamma$ increases.

Case (b) $\epsilon>\epsilon_{1}$; the chiral medium is denser than the dielectric. In this case, there is a change of handedness as $\theta_{i}$ passes through the Brewster angle. As $\gamma$ increases, $\left(b_{0} / a_{0}\right)_{\|}$ and $\left(b_{0} / a_{0}\right)_{\perp}$ become almost equal to zero; that is, the polarization ellipse becomes thin. Therefore, in this case, the reflected wave is almost linearly polarized.

\section{INFINITE CHIRAL SLAB}

In this section, plane-wave propagation through an infinite chiral slab of thickness $d$ is considered. The slab $(\epsilon, \mu, \gamma)$ is confined between two infinitely extended planes, $z=0$ and $z$ $=d$, and lies between two dielectrics with the same constitutive parameters $\left(\epsilon_{1}, \mu_{1}\right)$, as shown in Fig. 5. A plane wave is incident at an angle $\theta_{i}$ upon the chiral slab from the dielectric that borders the slab at $z=0$. The purpose of the analysis that follows is to find the amplitudes of the reflected and transmitted waves outside the slab. The incident electric and magnetic fields can be written as (see Fig. 5)

$$
\begin{aligned}
\mathbf{E}_{i} & =\mathbf{E}_{0 i} \exp \left[i k_{i}\left(z \cos \theta_{i}-x \sin \theta_{i}\right)\right], \\
\mathbf{H}_{i} & =\mathbf{H}_{0 i} \exp \left[i k_{i}\left(z \cos \theta_{i}-x \sin \theta_{i}\right)\right],
\end{aligned}
$$

where

$$
\begin{aligned}
& \mathbf{E}_{0 i}=E_{i \perp} \hat{e}_{y}+E_{i \|}\left(\cos \theta_{i} \hat{e}_{x}+\sin \theta_{i} \hat{e}_{z}\right), \\
& \mathbf{H}_{0 i}=\eta_{1}{ }^{-1}\left[E_{i \|} \hat{e}_{y}-E_{i \perp}\left(\cos \theta_{i} \hat{e}_{x}+\sin \theta_{i} \hat{e}_{z}\right)\right],
\end{aligned}
$$

and $\eta_{1}=\left(\mu_{1} / \epsilon_{1}\right)^{1 / 2}$. The reflected fields may be written as (see Fig. 5)

$$
\begin{aligned}
\mathbf{E}_{r} & =\mathbf{E}_{0 r} \exp \left[-i k_{i}\left(z \cos \theta_{i}+x \sin \theta_{i}\right)\right], \\
\mathbf{H}_{r} & =\mathbf{H}_{0 r} \exp \left[-i k_{i}\left(z \cos \theta_{i}+x \sin \theta_{i}\right)\right],
\end{aligned}
$$

where

$$
\begin{aligned}
& \mathbf{E}_{0 r}=E_{r \perp} \hat{e}_{y}+E_{r \|}\left(\cos \theta_{i} \hat{e}_{x}-\sin \theta_{i} \hat{e}_{z}\right), \\
& \mathbf{H}_{0 r}=\eta_{1}^{-1}\left[-E_{r \| \hat{e}_{y}}+E_{r \perp}\left(\cos \theta_{i} \hat{e}_{x}-\sin \theta_{i} \hat{e}_{z}\right)\right] .
\end{aligned}
$$

In the chiral slab, it is assumed that there are four total waves, two propagating toward the interface $z=d$ and the other two propagating toward the interface $z=0$ (see Fig. 5). The electric and magnetic fields of the two waves propagating inside the chiral medium toward the interface $z=d$ can be written as

$$
\begin{aligned}
\mathbf{E}_{c}{ }^{+}= & \mathbf{E}_{01}{ }^{+} \exp \left[i h_{1}\left(z \cos \theta_{1}-x \sin \theta_{1}\right)\right] \\
& +\mathbf{E}_{02}{ }^{+} \exp \left[i h_{2}\left(z \cos \theta_{2}-x \sin \theta_{2}\right)\right],
\end{aligned}
$$

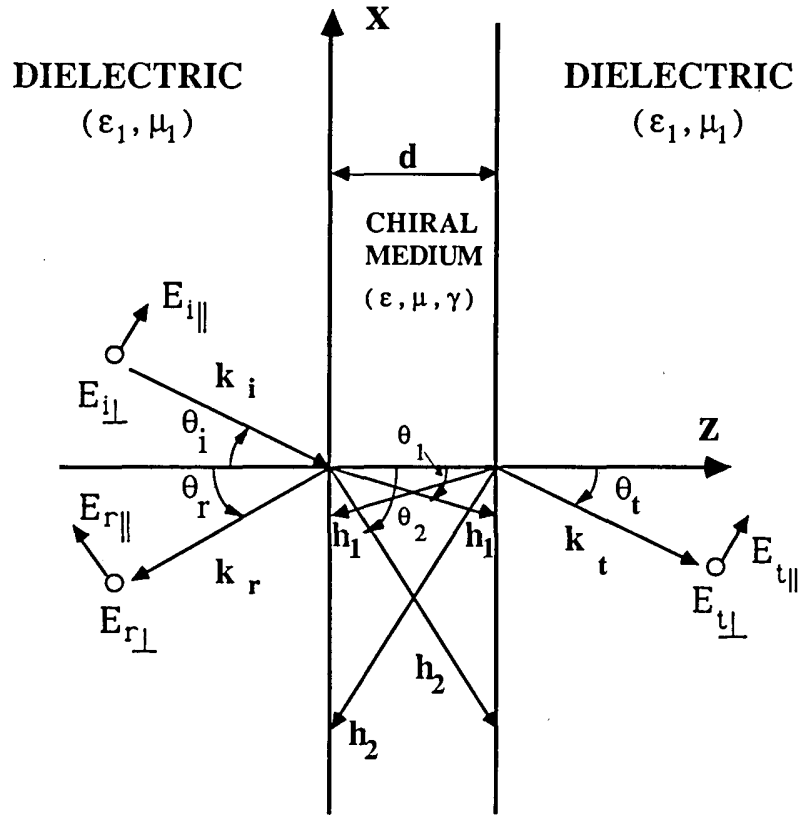

Fig. 5. Oblique incidence on an infinite slab of chiral medium. The dielectrics occupying the regions $z<0$ and $z>d$ have the same constitutive parameters. In the chiral slab, the $h_{1}$ and $h_{2}$ waves are right-circularly and left-circularly polarized waves, respectively.

$$
\begin{aligned}
\mathbf{H}_{c}{ }^{+}= & \mathbf{H}_{01}{ }^{+} \exp \left[i h_{1}\left(z \cos \theta_{1}-x \sin \theta_{1}\right)\right] \\
& +\mathbf{H}_{02}{ }^{+} \exp \left[i h_{2}\left(z \cos \theta_{2}-x \sin \theta_{2}\right)\right],
\end{aligned}
$$

where

$$
\begin{aligned}
& \mathbf{E}_{01}{ }^{+}=E_{01}{ }^{+}\left(\cos \theta_{1} \hat{e}_{x}+\sin \theta_{1} \hat{e}_{z}+i \hat{e}_{y}\right) \\
& \mathbf{H}_{01}{ }^{+}=-i Z^{-1} E_{01}{ }^{+}\left(\cos \theta_{1} \hat{e}_{x}+\sin \theta_{1} \hat{e}_{z}+i \hat{e}_{y}\right),
\end{aligned}
$$

and

$$
\begin{aligned}
& \mathbf{E}_{02}{ }^{+}=E_{02}{ }^{+}\left(\cos \theta_{2} \hat{e}_{x}+\sin \theta_{2} \hat{e}_{z}-i \hat{e}_{y}\right), \\
& \mathbf{H}_{02}{ }^{+}=i Z^{-1} E_{02}{ }^{+}\left(\cos \theta_{2} \hat{e}_{x}+\sin \theta_{2} \hat{e}_{z}-i \hat{e}_{y}\right) .
\end{aligned}
$$

The electric and magnetic fields of the other two total waves propagating inside the chiral medium toward the interface $z$ $=0$ may be written as

$$
\begin{aligned}
\mathbf{E}_{c}{ }^{-}= & \mathbf{E}_{01}{ }^{-} \exp \left[-i h_{1}\left(z \cos \theta_{1}+x \sin \theta_{1}\right)\right] \\
& +\mathbf{E}_{02}{ }^{-} \exp \left[-i h_{2}\left(z \cos \theta_{2}+x \sin \theta_{2}\right)\right], \\
\mathbf{H}_{c}{ }^{-}= & \mathbf{H}_{01}{ }^{-} \exp \left[-i h_{1}\left(z \cos \theta_{1}+x \sin \theta_{1}\right)\right] \\
& +\mathbf{H}_{02}{ }^{-} \exp \left[-i h_{2}\left(z \cos \theta_{2}+x \sin \theta_{2}\right)\right],
\end{aligned}
$$

where

$$
\begin{aligned}
& \mathbf{E}_{01}{ }^{-}=E_{01}{ }^{-}\left(\sin \theta_{1} \hat{e}_{z}-\cos \theta_{1} \hat{e}_{x}+i \hat{e}_{y}\right), \\
& \mathbf{H}_{01}{ }^{-}=-i Z^{-1} E_{01}{ }^{-}\left(\sin \theta_{1} \hat{e}_{z}-\cos \theta_{1} \hat{e}_{x}+i \hat{e}_{y}\right),
\end{aligned}
$$

and

$$
\begin{aligned}
& \mathbf{E}_{02}{ }^{-}=E_{02}{ }^{-}\left(\sin \theta_{2} \hat{e}_{z}-\cos \theta_{2} \hat{e}_{x}-i \hat{e}_{y}\right) \\
& \mathbf{H}_{02}{ }^{-}=i Z^{-1} E_{02}{ }^{-}\left(\sin \theta_{2} \hat{e}_{z}-\cos \theta_{2} \hat{e}_{x}-i \hat{e}_{y}\right)
\end{aligned}
$$

and the wave impedance $Z$ of the chiral medium is defined by $Z=(\mu / \epsilon)^{1 / 2}\left[1+(\mu / \epsilon) \gamma^{2}\right]^{-1 / 2}$. Outside the slab, in the 
dielectric that borders the slab at $z=d$, the total transmitted wave can be written as

$$
\begin{aligned}
\mathbf{E}_{t} & =\mathbf{E}_{0 t} \exp \left[i k_{t}\left(z \cos \theta_{t}-x \sin \theta_{t}\right)\right], \\
\mathbf{H}_{t} & =\mathbf{H}_{0 t} \exp \left[i k_{t}\left(z \cos \theta_{t}-x \sin \theta_{t}\right)\right],
\end{aligned}
$$

where

$$
\begin{aligned}
& \mathbf{E}_{0 t}=E_{t \perp} \hat{e}_{y}+E_{t \|}\left(\cos \theta_{t} \hat{e}_{x}+\sin \theta_{t} \hat{e}_{z}\right), \\
& \mathbf{H}_{0 t}=\eta_{1}^{-1}\left[E_{t \|} \hat{e}_{y}-E_{t \perp}\left(\cos \theta_{t} \hat{e}_{x}+\sin \theta_{t} \hat{e}_{z}\right)\right],
\end{aligned}
$$

where $k_{t}=k_{i}$ and $\theta_{t}=\theta_{i}$.

To find the complex-constant amplitude vectors of the reflected and transmitted waves in the two dielectrics and those of the waves inside the slab, the boundary conditions at the two interfaces $z=0$ and $z=d$ must be applied to the $x$ and $y$ components of the electric and magnetic fields. When this is done, a system of eight nonhomogeneous equations with the eight unknowns, $E_{r \perp}, E_{r \|}, E_{01}{ }^{+}, E_{02}{ }^{+}, E_{01}{ }^{-}, E_{02}{ }^{-}$, $E_{t \perp}$, and $E_{t \|}$, is obtained. This system of equations can be written in the following matrix form:

$$
\left[\begin{array}{c}
E_{r \perp} \\
E_{r \|} \\
E_{01}{ }^{+} \\
E_{02}{ }^{+} \\
E_{01}{ }^{-} \\
E_{02}^{-} \\
E_{t \perp} \\
E_{t \|}
\end{array}\right]=\mathbf{Q}^{-1}\left[\begin{array}{c}
E_{i \|} \\
E_{i \perp} \\
E_{i \perp} \\
E_{i \|} \\
0 \\
0 \\
0 \\
0
\end{array}\right],
$$

where $\mathbf{Q}$ is the following matrix:

$$
\mathbf{Q}=\left[\begin{array}{rrcc}
0 & -1 & R_{1} & R_{2} \\
-1 & 0 & i & -i \\
1 & 0 & i g R_{1} & -i g R_{2} \\
0 & 1 & g & g \\
0 & 0 & R_{1} \exp \left(i \delta_{1}\right) & R_{2} \exp \left(i \delta_{2}\right) \\
0 & 0 & i \exp \left(i \delta_{1}\right) & -i \exp \left(i \delta_{2}\right) \\
0 & 0 & -i g R_{1} \exp \left(i \delta_{1}\right) & i g R_{2} \exp \left(i \delta_{2}\right) \\
0 & 0 & g \exp \left(i \delta_{1}\right) & g \exp \left(i \delta_{2}\right)
\end{array}\right.
$$

$$
\mathbf{E}_{r}=E_{r} \hat{e}_{x} \exp \left(-i k_{i} z\right)
$$

where

$$
E_{r}=E_{i}\left(\frac{1+g}{1-g}\right) \frac{1-\exp \left[i\left(\delta_{1}+\delta_{2}\right)\right]}{[(1+g) /(1-g)]^{2}-\exp \left[i\left(\delta_{1}+\delta_{2}\right)\right]} .
$$

Therefore the polarization of the reflected wave is the same as that of the incident wave; that is, the chiral slab behaves as an ordinary dielectric as far as the polarization of the reflected wave is concerned. The transmitted wave can be written as

$$
\mathbf{E}_{t}=E_{t}\left[\hat{e}_{x}+\tan \left(\frac{\delta_{2}-\delta_{1}}{2}\right) \hat{e}_{y}\right] \exp \left(i k_{i} z\right),
$$

where

$$
E_{t}=E_{i} \frac{2 g}{(1-g)^{2}} \frac{\exp \left[i\left(\delta_{1}-\delta_{i}\right)\right]+\exp \left[i\left(\delta_{2}-\delta_{i}\right)\right]}{[(1+g) /(1-g)]^{2}-\exp \left[i\left(\delta_{1}+\delta_{2}\right)\right]} .
$$

Since $\tan \left[\left(\delta_{2}-\delta_{1}\right) / 2\right]$ is real, the transmitted wave is linearly polarized, and the ratio of its $x$ and $y$ components,

$$
\frac{E_{t y}}{E_{t x}}=\tan \left(\frac{\delta_{2}-\delta_{1}}{2}\right)=\tan (-\omega \mu \gamma d),
$$

shows that the plane of polarization of the transmitted wave is rotated by an angle of $-\omega \mu \gamma d$ with respect to the positive $x$ axis. If $\gamma$ is positive, then the rotation is toward the negative $y$ axis; if $\gamma$ is negative, then the rotation is toward the positive $y$ axis. where $R_{1}=\cos \theta_{1} / \cos \theta_{i}, R_{2}=\cos \theta_{2} / \cos \theta_{i}, \delta_{1}=h_{1} d \cos \theta_{1}, \delta_{2}=$ $h_{2} d \cos \theta_{2}$, and $\delta_{i}=k_{i} d \cos \theta_{i}$.

Since the analytical solution of this system of eight nonhomogeneous equations leads to involved expressions for the field amplitudes, it is therefore best to use numerical techniques to invert the matrix equation (66). However, it is interesting and important to obtain the analytical solution of this system for the case in which a linearly polarized wave is normally incident upon the interface. With no loss of generality, it is assumed that the incident electric field is directed along the positive $x$ axis. This field can be written as

$$
\mathbf{E}_{i}=E_{i} \hat{e}_{x} \exp \left(i k_{i} z\right)
$$

By setting $\theta_{i}$ and $E_{i \perp}$ to zero in the matrix equation (66) and then solving the resulting matrix equation, the reflected and transmitted electric fields can be found..$^{35}$ The reflected field can be written as

$\left.\begin{array}{cccc}-R_{1} & -R_{2} & 0 & 0 \\ i & -i & 0 & 0 \\ -i g R_{1} & i g R_{2} & 0 & 0 \\ g & g & 0 & 0 \\ -R_{1} \exp \left(-i \delta_{1}\right) & -R_{2} \exp \left(-i \delta_{2}\right) & 0 & -\exp \left(i \delta_{i}\right) \\ i \exp \left(-i \delta_{1}\right) & -i \exp \left(-i \delta_{2}\right) & -\exp \left(i \delta_{i}\right) & 0 \\ g R_{1} \exp \left(-i \delta_{1}\right) & -i g R_{2} \exp \left(-i \delta_{2}\right) & \exp \left(i \delta_{i}\right) & 0 \\ g \exp \left(-i \delta_{1}\right) & g \exp \left(-i \delta_{2}\right) & 0 & -\exp \left(i \delta_{i}\right)\end{array}\right]$

\section{CONCLUSIONS}

In this paper, the reflection from and transmission through a semi-infinite chiral medium are analyzed by obtaining the Fresnel equations in terms of parallel- and perpendicularpolarized modes and a set of symmetric constitutive relations. The results are compared with previous data, and the physical equivalence of the results is demonstrated. Also, the conditions for total internal reflection and for the Brewster angle are obtained. By using the Stokes parameters, the power carried by, and the polarization of, the reflected wave is studied. The problem of electromagnetic wave propagation through an infinite slab of chiral medium is formulated for oblique incidence and is solved analytically for the case of normal incidence. It is shown that, in this case, the plane of polarization of the transmitted wave is rotated with respect to that of the incident wave and that the plane of polarization of the reflected wave is unchanged with respect to that of the incident wave. 
The results of this paper have many potential applications in remote sensing and optics. In remote sensing, for instance, the vegetation layers can, in some cases, be modeled as chiral slabs. Thus the results obtained in this paper can be used to analyze radar data from such vegetation layers and to obtain the physical characteristics of the layers.

\section{ACKNOWLEDGMENT}

The authors thank Charles Elachi, the assistant laboratory director of the Office of Space Science and Instruments, Jet Propulsion Laboratory, California Institute of Technology, for his interest in this problem and for his kind assistance and advice.

\section{REFERENCES}

1. D. F. Arago, "Sur une modification remarquable qu' éprouvent les rayons lumineux dans leur passage à travers certains corps diaphanes, et sur quelques autres nouveaux phénomènes d'optique," Mem. Inst. 1, 93-134 (1811).

2. J. B. Biot, "Mémoire sur un nouveau genre d'oscillations que les molécules de la lumière éprouvent, en traversant certains cristaux," Mem. Inst. 1, 1-372 (1812).

3. J. B. Biot, "Sur les rotations que certaines substances impriment aux axes de polarisation des rayons lumineux," Mem. Acad. Sci. 2, 41-136 (1817).

4. J. B. Biot, "Mémoire sur la polarisation circulaire et sur ses applications à la chimie organique," Mem. Acad. Sci. 13, 39-175 (1835).

5. J. B. Biot, "Phénomènes de polarisation successive, observés dans des fluides homogènes," Bull. Soc. Philomath. 190-192 (1815).

6. A. Fresnel, "Mémoire sur la double réfraction que les rayons lumineux éprouvent en traversant les aiguilles de cristal de roche suivant des directions parallèles à l'axe," Oeuvres 1, 731751 (1822).

7. L. Pasteur, "Sur les relations qui peuvent exister entre la forme cristalline, la composition chimique et le sens de la polarisation rotatoire," Ann. Chim. Phys. 24, 442-459 (1848).

8. K. F. Lindman, "Über eine durch ein isotropes System von Spiralförmigen Resonatoren erzeugte Rotationspolarisation der elektromagnetischen Wellen," Ann. Phys. 63, 621-644 (1920).

9. K. F. Lindman, "Über die durch ein aktives Raumgitter erzeugte Rotationspolarisation der elektromagnetischen Wellen," Ann. Phys. 69, 270-284 (1922).

10. W. H. Pickering, California Institute of Technology, Pasadena, California 91125 (personal communication, 1945).

11. T. M. Lowry, Optical Rotatory Power (Dover, New York, 1964).

12. M. Born, "Über die natürliche optische Aktivität von Flüssigkeiten und Gasen," Phys. Z. 16, 251-258 (1915).

13. C. W. Oseen, "Über die Wechselwirkung zwischen zwei elektrischen Dipolen und über in Kristallen und Flüssigkeiten," Ann. Phys. 48, 1-56 (1915).
14. F. Gray, The optical activity of liquids and gases," Phys. Rev. 7, 472-488 (1916).

15. W. Kuhn, "Quantitative Verhältnisse und Beziehungen bei der natürlichen optischen Aktivität," Z. Phys. Chem. B 4, 14-36 (1929).

16. E. U. Condon, W. Altar, and H. Eyring, "One-electron rotatory power," J. Chem. Phys. 5, 753-775 (1937).

17. E. U. Condon, "Theories of optical rotatory power," Rev. Mod. Phys. 9, 432-457 (1937).

18. C. F. Bohren, "Light scattering by an optically active sphere," Chem. Phys. Lett. 29, 458-462 (1974).

19. C. F. Bohren, "Scattering of electromagnetic waves by an optically active cylinder," J. Colloid Interface Sci. 66, 105-109 (1975).

20. B. V. Bokut and F. I. Federov, "Reflection and refraction of light in optically isotropic active media," Opt. Spektrosk. 9, 334-336 (1960).

21. J. A. Kong, Theory of Electromagnetic Waves (Wiley, New York, 1975).

22. D. L. Jaggard, A. R. Mickelson, and C. H. Papas, "On electromagnetic waves in chiral media," Appl. Phys. 18, 211-216 (1979).

23. N. Engheta and A. R. Mickelson, "Transition radiation caused by a chiral plate," IEEE Trans. Antennas Propag. AP-30, 12131216 (1982).

24. M. P. Silverman, "Reflection and refraction at the surface of a chiral medium: comparison of gyrotropic constitutive relations invariant or noninvariant under a duality transformation," J. Opt. Soc. Am. A 3, 830-837 (1986).

25. M. P. Silverman, "Specular light scattering from a chiral medium: unambiguous test of gyrotropic constitutive relations," Lett. Nuovo Cimento 43, 378-382 (1985).

26. A. Lakhtakia, V. V. Varadan, and V. K. Varadan, "A parametric study of microwave reflection characteristics of a planar achiral-chiral interface," IEEE Trans. Electromagn. Compat. EM-28, 90-95 (1986).

27. A. Lakhtakia, V. V. Varadan, and V. K. Varadan, "Scattering and absorption characteristics of lossy dielectric, chiral, nonspherical objects," Appl. Opt. 24, 4146-4154 (1985).

28. M. P. Silverman and R. B. Sohn, "Effects of circular birefringence on light propagation and reflection," Am. J. Phys. 54, 6976 (1986).

29. S. Bassiri, N. Engheta, and C. H. Papas, "Dyadic Green's function and dipole radiation in chiral media," Alta Freq. 2, 83-88 (1986).

30. S. Bassiri, "Electromagnetic wave propagation and radiation in chiral media," doctoral dissertation (Division of Engineering and Applied Science, California Instititute of Technology, Pasadena, Calif., 1987).

31. A. Sommerfeld, Optics (Academic, New York, 1954).

32. M. Born and E. Wolf, Principles of Optics (Pergamon, New York, 1980).

33. C. H. Papas, Theory of Electromagnetic Wave Propagation (McGraw-Hill, New York, 1965).

34. H. C. Chen, Theory of Electromagnetic Waves (McGraw-Hill, New York, 1983).

35. J. A. Stratton, Electromagnetic Theory (McGraw-Hill, New York, 1941). 